\title{
Attentional Orienting Is Impaired by Unilateral Lesions of the Thalamic Reticular Nucleus in the Rat
}

\author{
G. Daniel Weese, ${ }^{1}$ Janice M. Phillips, ${ }^{2}$ and Verity J. Brown ${ }^{2}$ \\ ${ }^{1}$ Department of Psychology, Hampden-Sydney College, Hampden-Sydney, Virginia 23943, and 2School of Psychology, \\ University of St. Andrews, St. Andrews KY16 9JU, Scotland, United Kingdom
}

The thalamic reticular nucleus (TRN) has been implicated in attentional processes based on its anatomical, electrophysiological, and neurochemical relationships with the sensory nuclei of the thalamus and corresponding sensory areas of cortex. This study examined the possibility that the TRN is involved in covert orienting of attention. Attention can be summoned to a spatial location in the absence of an overt orienting response. The reaction time to a visual target is faster when attention has been drawn to the location of the target by a preceding cue in that location (valid cue) compared with when the cue misdirects attention (invalid cue) away from the location of the subsequent target. This reaction time difference is referred to as the "validity effect."

A role for the thalamic reticular nucleus (TRN) in selective attention is suggested by studies of its anatomical, electrophysiological, and neurochemical interactions with the sensory nuclei of the dorsal thalamus and their respective cortical areas (Guillery et al., 1998). Thus, Yingling and Skinner (1977) wrote that the thalamic reticular nucleus is "an obvious candidate for inclusion in any proposed control mechanism in the brain that underlies functions such as 'attention'." The TRN is a sheet of GABAergic cells to which both thalamocortical and corticothalamic fibers send collaterals (Jones, 1985; Ohara and Lieberman, 1985). Within the TRN, signals are segregated by sensory modality, with the visual sector located dorsally and caudally in the nucleus (Coleman and Mitrofanis, 1996; Loszadi et al., 1996).

Activity in the TRN may gate the thalamocortical flow of information by sharpening receptive fields and response times of thalamic neurons (Lee et al., 1994; Liu et al., 1995; Cox et al., 1997; Kim et al., 1997; Cox and Sherman, 1999) and modulating cortical arousal (Block, 1994; McDonald et al., 1998). Although there is physiological evidence for an attentional role of the TRN (Steriade et al., 1986), the behavioral evidence bearing on the issue is inconclusive. Exposure to a novel complex visual environment induced immediate early gene expression in visual TRN (Montero, 1997), and asymmetry of exploratory behavior results in asymmetric Fos-labeling in TRN (Montero, 1999). Lesions of the TRN produce multimodal deficits in orientation (Friedberg

\footnotetext{
Received June 7, 1999; revised Aug. 18, 1999; accepted Sept. 3, 1999.

This work was supported by The Wellcome Trust Project Grant 051945/Z/97/Z We thank Dr. C. Davidson for assistance, M. Latimer for performing the histology, and the animal care staff of the School of Psychology Animal House and the workshop technicians, particularly Pete Wilcox for the maintenance of equipment.

Correspondence should be addressed to Dr. Verity J. Brown, School of Psychology, University of St. Andrews, St. Andrews KY16 9JU, Scotland, UK. E-mail: vjb@st-and.ac.uk.

Copyright (C) 1999 Society for Neuroscience 0270-6474/99/1910135-05\$05.00/0
}

Rats were trained to perform such a reaction time task with visual cues and targets presented in poke holes to either side of the rat's head, which had to be maintained centrally and still. If the rat made an overt orienting response to the cue, the trial was aborted. Unilateral lesions were made by injection of ibotenic acid in the TRN. After surgery, there was no bias apparent in their responding; they were as likely to initiate responses and were equally accurate to either side. There was, however, a complete abolition of the validity effect for responses to contralateral targets. The data are discussed in terms of a role for the TRN in attentional processing.

Key words: attention; orienting; neglect; thalamic reticular nucleus; ibotenic acid; rat

and Ross, 1993), but these tests of overt orientation also do not unequivocally demonstrate attentional rather than a sensory or motor role (Hoyman et al., 1979; Carli et al., 1985; Brown and Robbins, 1989). The TRN may have a role in learning and memory (M'Harzi et al., 1991; Collery et al., 1993; Tenas-Huerga et al., 1998), but such function neither demonstrates nor rules out involvement in attentional processes.

Posner (1980) devised a laboratory task to measure covert attentional processes, which has enabled the neural and pharmacological substrates of attention to be explored (Posner et al., 1984; Petersen et al., 1987; Robinson et al., 1995; Witte et al., 1997). A cue that precedes a target in the same location speeds reaction time to the target compared with a cue that misdirects attention to a different location. This reaction time effect is thought to reflect the benefit of directed attention and/or the cost of misdirected attention. Administration of GABAergic compounds into the pulvinar of monkeys modifies shifts of attention (Petersen et al., 1987). The pulvinar receives GABAergic projections from the TRN and from the superior colliculus (Lane et al., 1997), either or both of which may be significant for attention (Robinson and Kertzman, 1995).

It is possible to measure covert orienting in the rat (Ward and Brown, 1996), and evidence suggests that the TRN is a likely candidate as the substrate of covert orienting. Therefore, in the present study, we explored the effects of lesions of the TRN on covert orienting in the rat.

Parts of the paper have been published previously in abstract form (Weese and Brown, 1998).

\section{MATERIALS AND METHODS}

Animals. Twenty Lister hooded rats (Charles River, Kent, UK) were housed individually in $25 \times 45 \times 15 \mathrm{~cm}$ plastic cages on a $12 \mathrm{hr}$ light/dark cycle. Testing was conducted in the light phase. The rats were maintained on a restricted diet of 15-20 gm of food (reward pellets plus standard 
laboratory chow) per day, and ad libitum water was available in the home cage throughout. The weight range was $215-280 \mathrm{gm}$ at the start and 373-432 gm at completion of the study. The guidelines laid out in the Principles of Laboratory Animal Care (National Institutes of Health, Publication No. 86-23, revised 1985) and the requirements of the United Kingdom Animals (Scientific Procedures) Act 1986, were carefully followed throughout.

Apparatus. The test apparatus was the same nine-hole box $(\mathrm{CeNeS}$, Cambridge, UK) used in a study of covert orienting by Ward and Brown (1996). Inset into one wall of the chamber is an array of poke holes. At the rear of each hole is a single bulb that can be illuminated at varying levels of brightness. Photocell light source assemblies across the front of each hole detect nose pokes into the hole. In the wall opposite the response holes is a food hopper occluded by a hinged panel and containing a light. An automatic dispenser delivers pellets $(45 \mathrm{mg}$; BioServ, Frenchtown, NJ) to the hopper. The chamber is illuminated by a house light in the center of the ceiling, and the entire chamber was encased in a ventilated sound-attenuating box.

Training regimen. The training and testing protocols were essentially the same as that described by Ward and Brown (1996). First, rats were habituated to the chamber for $1 \mathrm{hr}$ with food pellets placed in the hopper. Second, they were trained to collect food from the hopper over several days by making food delivery and light onset in the hopper contingent on a panel press. Third, a trial was initiated by a panel press, which turned off the hopper light and illuminated the lamp in the center hole. Nose pokes into the center hole were rewarded by the onset of the hopper lamp and the delivery of a pellet. The required duration of the nose poke was gradually increased over days; if a rat failed to maintain the required nose poke duration, a $1 \mathrm{sec}$ "time-out" period ensued, during which the house light was extinguished and no food was available. Then, the house and hopper lights turned on, and the rat could initiate another trial with a panel press. In the fourth and final stage, the testing paradigm was introduced.

Testing paradigm. The trial events are depicted in Figure 1. A trial was initiated by the rat pushing the hopper panel, which resulted in the illumination of the central hole. A nose poke into the center hole started a foreperiod of $0.2,0.3,0.4$, or $0.5 \mathrm{sec}$, at the end of which a brightly lit target appeared in one of the side holes with a duration of $150 \mathrm{msec}$. At the start of the foreperiod, a dim cue light was illuminated for $100 \mathrm{msec}$ in either one of the side holes. On $50 \%$ of the trails, the cue was presented in the location of the subsequent target (i.e., a valid cue); invalid cues (appearing on the side opposite the subsequent target) were presented on the remaining $50 \%$ of the trials. The order of valid and invalid cue trials and variable foreperiods was randomized. A correct response into the target hole, within $2 \mathrm{sec}$ of withdrawing from the center hole, was rewarded with a food pellet. Errors (see below) caused the house light to be extinguished for a $1 \mathrm{sec}$ time-out and were not followed by a reward. Although trials were not repeated after an error, when the rat made an anticipatory withdrawal before target onset, the subsequent trial used the same foreperiod with only the side of the cue and the target being reselected. This was to avoid the possibility that the rat would abort all the trials with the longest foreperiods.

The eyes of a rat are lateral in its head and thus both cannot be directed to one side only. Exploratory eye movements are thought to be unlikely in the rat (Sefton and Dreher, 1995) but, rather, orienting head movements are made toward stimuli of interest. Therefore, for confirmation of covert orienting of attention in the rat, it is most important that the head of the rat is maintained centrally and still. The configuration of the poke holes is such that, when the rat makes a sustained nose poke, its head is oriented forward and level. Any processing enhancement of the target conferred by a peripheral cue cannot therefore be attributable to previous foveation of (i.e., an overt orientation to) the target location but must be caused by a shift in attention.

Testing sessions were terminated after completion of 120 correct trials. Presurgical testing continued until the rat demonstrated stable reaction times and statistically significant shorter reaction times to targets preceded by a valid cue than when preceded by an invalid cue over five test sessions (600 trials). Postsurgical testing began after a $2 \mathrm{~d}$ recovery period and lasted for $5 \mathrm{~d}$ (600 trials).

Definition of measures. Reaction time was the time from target onset to withdrawal from the central hole. Three types of errors were possible: a nose poke in the hole opposite the target was an incorrect response; withdrawal from the central hole before the onset of the target was an anticipatory error; and failure to respond to either side hole within $2 \mathrm{sec}$ was a late error.

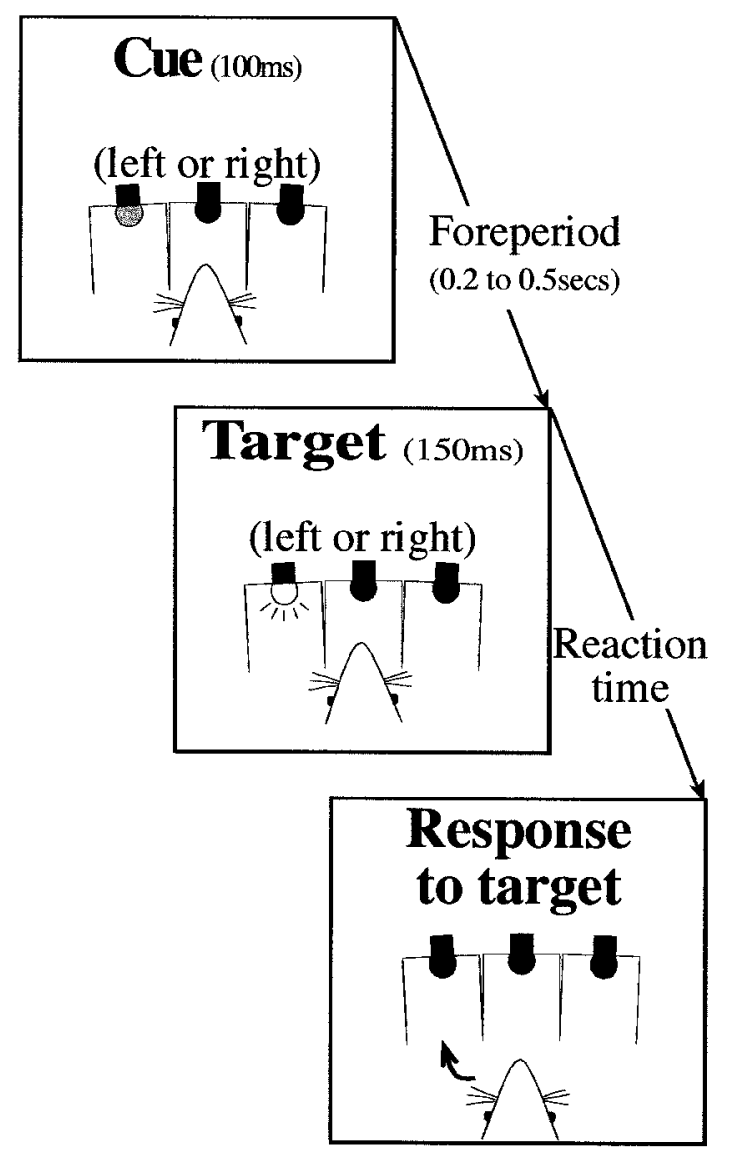

Figure 1. Schematic illustration of trial events. Trials were initiated by the rat making a sustained nose poke into the central of three holes. At the start of the variable foreperiod, a cue was presented to the left or right. The target could also appear on the left or the right. The side of the cue did not predict the side of the target; for $50 \%$ of trials, the target was on the same side as the cue (validly cued), and for the remaining $50 \%$, it was on the opposite side (invalidly cued). The rat reported the location of the target by making a nose poke response in the side hole.

Surgery. The rats received a unilateral infusion of the neurotoxin ibotenic acid (Tocris Cookson, Bristol, UK) into the TRN. The side of the lesion was assigned randomly.

Anesthesia was induced with an intraperitoneal injection of pentobarbitone sodium $(1.0 \mathrm{ml} / \mathrm{kg}, 65 \mathrm{mg} / \mathrm{ml})$. The rats were then placed in a stereotaxic frame with atraumatic ear bars (Kopf, Tujunga, CA), with the nose bar set at $+5 \mathrm{~mm}$. A midline incision was made along the scalp, and the skin and fascia were retracted to expose the skull. A hole was then drilled in the skull on one side only, at the coordinates of $-3.6 \mathrm{~mm}$ posterior and $\pm 3.8 \mathrm{~mm}$ lateral to bregma. The needle of a $1 \mu \mathrm{l}$ syringe containing $0.3 \mu \mathrm{l}$ of $0.09 \mathrm{M}$ ibotenic acid was then lowered to $-7.4 \mathrm{~mm}$ below the skull surface, and the ibotenic acid was infused manually at a rate of $0.1 \mu \mathrm{l}$ every $3 \mathrm{~min}$. The syringe was left in place for $3 \mathrm{~min}$ before being withdrawn slowly. The incision in the scalp was then closed using sterile metal clips. Finally, the animal was placed in a warm cage to recover before transfer back to a home cage.

Histology. At the conclusion of postsurgical testing, the rats were killed by intraperitoneal administration of Euthanol $(1.0 \mathrm{ml} / \mathrm{kg}$; pentobarbitone sodium, $200 \mathrm{mg} / \mathrm{ml}$ ). The rats were perfused transcardially with phosphate buffer for $2 \mathrm{~min}$ at a rate of $10 \mathrm{ml} / \mathrm{min}$, followed by a $4 \%$ paraformaldehyde in phosphate buffer for $20 \mathrm{~min}$ at the same rate. The brains were then removed and placed into a $20 \%$ sucrose $-4 \%$ paraformaldehyde phosphate buffer solution until processed. Serial coronal sections $50-\mu \mathrm{m}$-thick were cut using a freezing microtome, and two adjacent sections every $400 \mu \mathrm{m}$ were taken for staining with cresyl violet and immunohistochemistry with parvalbumin stain. The intact TRN stains darkly with parvalbumin, which can be used to assess cell loss in 

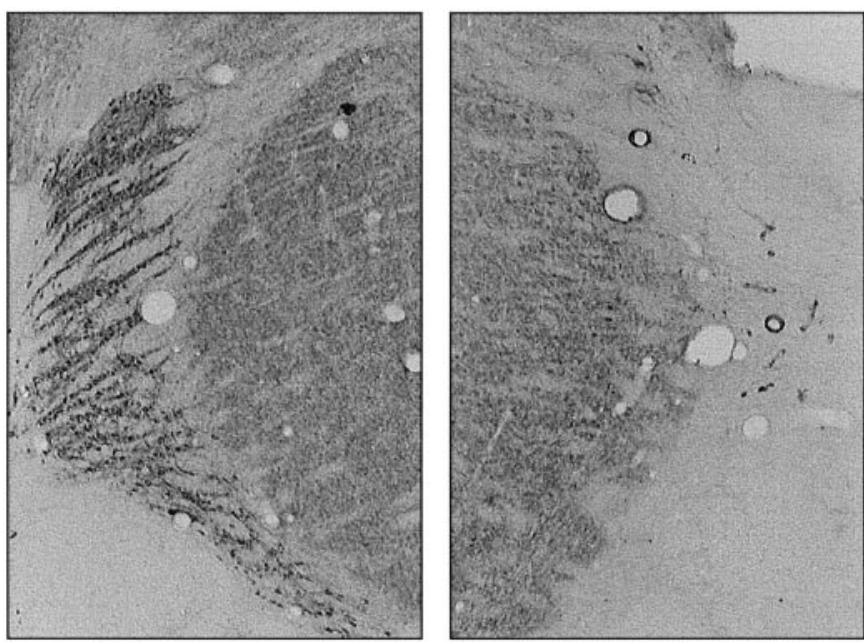

Figure 2. Parvalbumin-stained coronal sections at approximately bregma $-3.4 \mathrm{~mm}$ from the brain of one rat. The unlesioned TRN is shown in the left, and the lesioned tissue is shown on the right. All sectors of the TRN between coronal levels -3.0 and $-3.8 \mathrm{~mm}$ were lesioned in most rats. The TRN was spared anterior to bregma $-2.2 \mathrm{~mm}$.

that structure. Cresyl violet sections were also examined under a light microscope for evidence of damage outside of the TRN.

Data analysis. The reaction times and percentage of incorrect responses were compared for trials on which targets were presented ipsilateral or contralateral to the lesion. Repeated-measures ANOVA was used with four within-subject factors: surgery (preoperative and postoperative); side of target relative to lesion (ipsilateral and contralateral); cue type (valid and invalid); and duration of foreperiod (four levels: 0.2, $0.3,0.4$, and $0.5 \mathrm{sec}$ ). Analyses of simple main effects (Winer, 1971) were used to examine the source of multifactorial interactions. Anticipatory responses were also analyzed by ANOVA, but because these responses are made before the target, it is not meaningful to consider them in terms of side of target or cue validity. Therefore, these data were analyzed with only the within-subject factors of surgery (preoperative and postoperative) and foreperiod (four levels: 0.2, 0.3, 0.4, and $0.5 \mathrm{sec}$ ).

\section{RESULTS}

\section{Histology}

Fourteen rats sustained unilateral damage that included the visual sector of TRN. Of the six rats excluded from the analyses, one sustained a large lesion that encroached into the lateral geniculate nucleus, one was found to have a tumor in the thalamus, apparently unrelated to the lesioning procedure, and four had incomplete or no lesions. Figure 2 shows a coronal parvalbumin-stained section from the brain of one rat at approximately bregma $-3.4 \mathrm{~mm}$. The lesions typically extended from bregma $-2.6 \mathrm{~mm}$ to bregma $-3.8 \mathrm{~mm}$. In most cases, the lesion included auditory, as well as visual, TRN, typically with no sparing of the nucleus in the sections posterior to bregma -3.4 $\mathrm{mm}$. There was no obvious damage to the fiber tracts that border the TRN. There was no reduction in tissue volume to suggest loss of fibers, but we cannot rule out the possibility that demyelination might have occurred. It is possible that transient demyelination accompanies all excitotoxic lesions (Brace et al., 1997). There appears to be no method by which demyelination can be prevented; however, Brace et al. (1997) report the presence of axons within their excitotoxic lesion area, and the process of remyelination that they report is strongly suggestive that these fibers are functional.

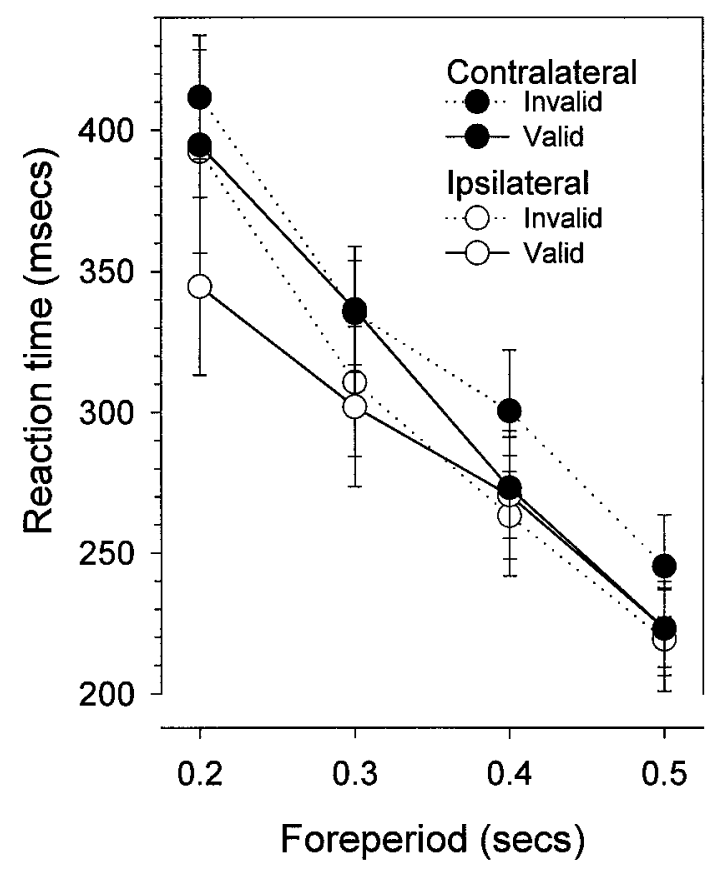

Figure 3. This figure shows the mean \pm SEM reaction time of 14 rats who had sustained unilateral lesions of the TRN. For responses to ipsilateral targets, reaction time is faster for validly cued trials at the shortest foreperiod. For reaction time to contralateral targets, there is no reaction time difference between validly and invalidly cued trials.

\section{Performance accuracy}

Overall, performance accuracy was over $90 \%$, and therefore there were too few error trials on which to base reliable conclusions regarding changes after the lesion. There were no reliable patterns in the frequency of late responses. Anticipatory responses increased as a function of foreperiod (main effect of foreperiod, $F_{(3,39)}=76.7 ; p<0.01$ ), as would be expected. After the lesion, there were fewer anticipatory responses (interaction of surgery and foreperiod, $\left.F_{(3,39)}=6.0 ; p<0.01\right)$. Incorrect responses were more likely at the shortest foreperiod after invalid cues (interaction of validity and foreperiod, $\left.F_{(3,39)}=3.4 ; p<0.05\right)$. However, these errors were infrequent, and there was no statistically significant change in the pattern of errors after the lesion (interaction of surgery, side, and validity, $F_{(3,39)}=0.005$; NS; interaction of surgery, side, validity, and foreperiod, $F_{(3,39)}=0.12$; NS).

\section{Reaction time}

The effects of the lesion were seen in the reaction time data; the reaction time difference between validly and invalidly cued targets (the validity effect) was abolished on the contralateral side. Figure 3 shows preoperative and postoperative reaction time plotted by side (ipsilateral and contralateral) and foreperiod. From the graphs, it is apparent that reaction time decreases as a function of foreperiod (main effect of foreperiod, $F_{(3,36)}=190.7 ; p<0.01$ ), and at the shortest foreperiod, reaction time is faster when preceded by a valid cue (interaction of validity and foreperiod, $\left.F_{(3,36)}=15.2 ; p<0.01\right)$. However, this pattern changed postoperatively; reaction time was no longer faster to contralateral targets when preceded by contralateral cues (interaction of surgery, side, validity, and foreperiod, $\left.F_{(3,36)}=3.9 ; p<0.02\right)$. Figure 4 shows the same data replotted to show the validity effect (invalid minus valid reaction time). The validity effect is significantly reduced at the shortest foreperiod in the postoperative data on 


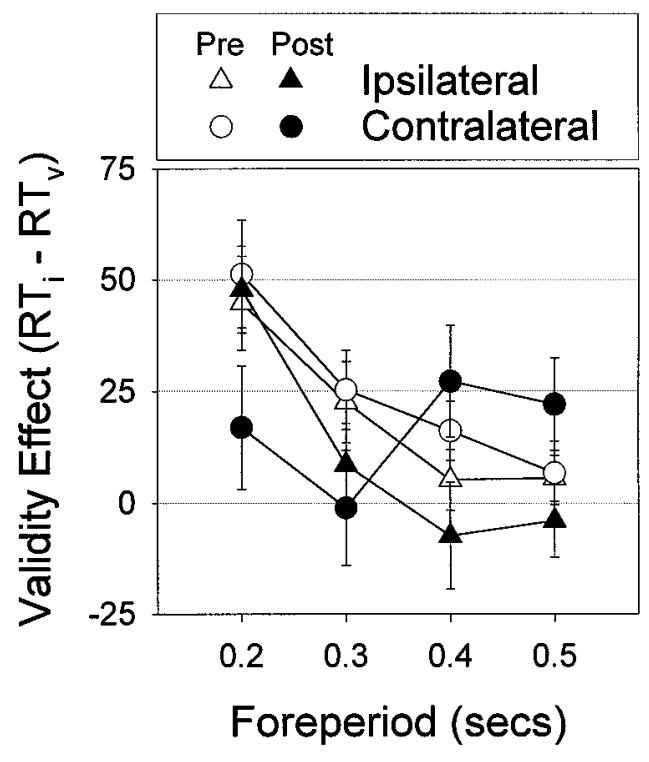

Figure 4. This figure shows the mean \pm SEM validity effect (invalid minus valid reaction time), preoperatively and postoperatively, for ipsilateral and contralateral responses. Preoperatively, the validity effect is equal for responses to either side. The validity effect is not seen in the postoperative data on the contralateral side, indicating an abolition of the benefit of attentional orienting to contralateral cues. There was no change in the magnitude of the validity effect on the ipsilateral side.

the contralateral side, indicating an abolition of the benefit of attentional orienting to contralateral cues (analysis of the validity effect restricted by side: interaction of surgery and foreperiod, $\left.F_{(3,39)}=4.5 ; p<0.01\right)$. The validity effect on the ipsilateral side was not significantly different (interaction of surgery and foreperiod, $\left.F_{(3,39)}=0.5 ; \mathrm{NS}\right)$.

\section{DISCUSSION}

In this study, we have shown that, after unilateral lesions of the TRN, there is no processing advantage for a contralateral target when it is preceded by a cue in the same location (a valid cue). Postoperatively, reaction times to validly cued contralateral targets increased to the level of invalidly cued targets, indicating that the contralateral cue no longer drew attention. Thus, the effect of the lesion was to abolish the processing advantage conferred by cues contralateral to the lesion. This is unequivocal evidence of a role for the TRN in covert orienting of attention.

To date, there have been few behavioral studies examining the effects of lesions of the TRN. This is most likely because of two factors: the anatomical position and configuration of the nucleus and the availability of suitable behavioral tests. The Discussion will first consider the former and then focus on the latter.

\section{Lesion specificity}

The anatomical position and configuration of the TRN in the rat and the primate is such that it would seem to be rather difficult to make a selective lesion. A lesion of the lateral geniculate nucleus would result in blindness and must therefore be avoided. Furthermore, the TRN forms a relatively thin sheet of tissue, interposed between the thalamus and cortex. It surrounds the thalamus, so it would appear that multiple small injections would be necessary to effect a lesion of sufficient size but that did not encroach into the cortex or thalamus. However, the TRN is bounded by the internal capsule and the medial lemniscus. We found that it was possible to make a lesion of the TRN, taking advantage of the fact that the injected neurotoxin spreads along the boundary fibers. The toxin "wicked" through the nucleus, resulting in a reasonably extensive and yet rather selective lesion (Fig. 2). Not all the injection placements were effective; even a small error of placement would result in an injection into fibers and an incomplete or no lesion. However, when an injection was placed in the TRN, the lesion was confined to TRN and did not include adjacent structures.

\section{Behavioral analysis}

The hypothesis that the TRN is the substrate of the spotlight of attention can only be tested using a sophisticated visual attentional task in which the ability to orient attention (i.e., covert orientation) can be distinguished from the ability to orient the head (i.e., overt orientation). Until recently, such a task has not been available for the rat. However, using this cued visual reaction time task, a great deal can be learned about orienting of attention and the role of the TRN. The pattern of reaction time changes after the lesion provides a good deal of additional information concerning the nature of the contribution of the TRN to covert orienting, and it is on this that the Discussion will focus.

When attention is directed to a spatial location, reaction time to a target is faster compared with when attention is misdirected (for example, away from the target location) before target onset. This could be attributable to a benefit for directing attention to the location of the target, to a cost of misdirecting attention, or to a combination of both of these factors. From the present results, it is apparent that, after the lesion, the benefit for processing of contralateral targets conferred by the contralateral cue is abolished. If there were a reduction of the cost of a cue presented contralaterally, one would expect a decrease in reaction times to the subsequent ipsilateral target. This decreased reaction time would reduce the validity effect on the ipsilateral side. If, on the other hand, there were a reduction of the benefit of a contralateral cue, an increase in reaction times to the contralateral target that it preceded would be expected. This increase would reduce or eliminate the validity effect on the contralateral side. If the lesion reduced the ability of a contralateral cue to both distract the animal from an ipsilateral target and prepare it for the presentation of a contralateral target, the validity effect would be reduced on both sides. After a unilateral lesion, we find changes in the validity effect for contralateral targets only. There is no reaction time advantage provided by valid cues presented on the side contralateral to the lesion, indicating that directed attention no longer benefits the processing of targets on the contralateral side. However, there is no corresponding reduction in cost when an ipsilateral target follows the contralateral cue. The evidence for a decrease in the benefit of a contralateral cue preceding a contralateral target, but not in the cost of an contralateral cue preceding an ipsilateral target, suggests that the effect of a peripheral cue is to prime the visual system to process a subsequent target in the same hemifield. A cue presented to the hemifield opposite the target is not a distraction because the transmission pathway for the ipsilateral target is unaffected.

Yingling and Skinner (1977) and Skinner and Yingling (1977) suggested that the TRN might be the neural substrate of an attentional filter that gates the flow of information to the cortex by means of its topographically organized inhibitory influence on thalamic relay nuclei. The cortex, in turn, regulates the influence of the TRN on thalamocortical transmission. Thus, the cortex determines which signals will be suppressed and which will be enhanced by the TRN. When a stimulus (for example, the cue in the present study) is presented, the glutamatergic synapses from 
the cortex onto TRN neurons cause the depolarization or disinhibition of thalamocortical neurons, possibly by stimulating the TRN inhibition of local-circuit GABAergic neurons in thalamic nuclei (Steriade et al., 1986) or by group II glutamate receptor mediated-inhibition of TRN efferent neurons (Cox and Sherman, 1999). The cue facilitates the transmission of a signal from any subsequent stimulus (such as the target) in the same field. The validity effect dissipates over time, corresponding to the maximum duration of the depolarization. A unilateral lesion in the TRN eliminates the priming effect of contralateral cues, resulting in an increase in reaction time to contralateral targets because they no longer benefit from this priming. When cues and targets are both ipsilateral, reaction time continues to show the benefit of priming because that pathway is unaffected by the lesion. This account fits well with the biased competition model of Desimone and Duncan (1995). This model states that stimuli compete for limited information processing resources and that a cue sets up an attentional template that biases the competition in favor of a target with the same features or in the same location. The TRN may play a role in the formation or maintenance of the attentional template.

In summary, the benefit of a valid cue on the reaction time to a visual target is found across species, and the present results provide new evidence that the TRN is critically involved in this process.

\section{REFERENCES}

Block F (1994) Stimulation of $N$-methyl-D-aspartate receptors in the rat nucleus reticularis thalami suppresses somatosensory evoked potentials. Brain Res 636:143-146.

Brace H, Latimer M, Winn P (1997) Neurotoxicity, blood-brain barrier breakdown, demyelination and remyelination associated with NMDAinduced lesions of the rat lateral. Brain Res Bull 43:447-455.

Brown VJ, Robbins TW (1989) Elementary processes of response selection mediated by distinct regions of the striatum. J Neurosci 9:3760-3765.

Carli M, Evenden J, Robbins TW (1985) Depletion of unilateral striatal dopamine impairs initiation of contralateral actions and not sensory attention. Nature 31:679-682.

Coleman KA, Mitrofanis J (1996) Oganization of the visual reticular thalamic nucleus of the rat. Eur J Neurosci 8:388-404.

Collery M, M'Harzi M, Delacour J (1993) Lesions of reticularis thalamic nucleus impair spatial working memory in rats. Neurosci Res Commun 12:41-49.

Cox CL, Sherman SM (1999) Glutamate inhibits thalamic reticular neurons. J Neurosci 19:6694-6699.

Cox CL, Huguenard JR, Prince DA (1997) Nucleus reticularis neurons mediate diverse inhibitory effects in thalamus. Proc Natl Acad Sci USA 94:8854-8859.

Desimone R, Duncan J (1995) Neural mechanisms of selective visual attention. Annu Rev Neurosci 18:193-222.

Friedberg EB, Ross DT (1993) Degeneration of rat thalamic reticular neurons following intrathalamic demoic acid injection. Neurosci Lett 151:115-119.

Guillery RW, Feig SL, Lozsadi DA (1998) Paying attention to the thalamic reticular nucleus. Trends Neurosci 21:28-32.

Hoyman L, Weese GD, Frommer GP (1979) Tactile discrimination deficits following neglect-producing unilateral hypothalamic lesions in the rat. Physiol Behav 22:139-147.

Jones EG (1985) The thalamus. New York: Plenum.

Kim U, Sanchez-Vives MV, McCormick DA (1997) Function dynamics of GABAergic inhibition in the thalamus. Science 278:130-134.
Lane RD, Allan DM, Bennett-Clark CA, Howell DL, Rhoades RW (1997) Projection status of calbindin- and parvalbuminimmunoreactive neurons in the superficial layers of the rats superior colliculus. Vis Neurosci 14:277-286.

Lee SM, Friedberg MH, Ebner FF (1994) The role of GABA-mediated inhibition in the rat ventral posterior medial thalamus: assessment of receptive field changes following thalamic reticular nucleus lesions. J Neurophysiol 71:1702-1715.

Liu XB, Warren RA, Jones EG (1995) Synaptic distribution of afferents from reticular nucleus in ventroposterior nucleus of cat thalamus. J Comp Neurol 352:187-202.

Lozsadi DA, Gonzales J, Guillery RW (1996) The course and termination of corticothalamic fibres arising in the visual cortex of the rat. Eur J Neurosci 8:2416-2427.

McDonald KD, Fifkova E, Jones MS, Barth DS (1998) Focal stimulation of the thalamic reticular nucleus induces focal gamma waves in cortex. J Neurophysiol 79:474-477.

M'Harzi M, Collery M, Delacour J (1991) First evidence of a possible role of the reticular thalamic nucleus in working memory in rats. Neurosci Res Commun 8:167-174.

Montero VM (1997) C-fos induction in sensory pathways of rats exploring a novel complex environment: shifts of active thalamic reticular sectors by predominant sensory cues. Neuroscience 76:1069-1081.

Montero VM (1999) Amblyopia decreases activation of the corticogeniculate pathway and visual thalamic reticularis in attentive rats: a "focal attention" hypothesis. Neuroscience 91:805-817.

Ohara PT, Lieberman AR (1985) The thalamic reticular nucleus of the adult-rat-experimental anatomical studies. J Neurocytol 14:365-411.

Petersen SE, Robinson DL, Morris JD (1987) The contribution of the pulvinar to visual spatial attention. Neuropsychologia 25:97-105.

Posner MI (1980) Orienting of attention. Q J Exp Psychol 32:3-25.

Posner MI, Walker JA, Friedrich FJ, Rafal RD (1984) Effects of parietal injury on covert orienting of attention. J Neurosci 4:1863-1874.

Robinson DL, Kertzman C (1995) Covert orienting of attention in Macaques. III. Contributions of the superior colliculus. J Neurophysiol 74:713-721.

Robinson DL, Bowman EM, Kertzman C (1995) Covert orienting of attention in macaques. II. Contributions of parietal cortex J Neurophysiol 74:698-712.

Sefton AJ, Dreher B (1995) Visual system. In: The rat nervous system, Ed 2 (Paxinos G, ed). New York: Academic.

Skinner JE, Yingling CD (1977) Reconsideration of the cerebral mechanisms underlying selective attention and slow potential shifts. In: Attention, voluntary contraction and event-related cerebral potentials. Progress in clinical neurophysiology, Vol 1 (Desmedt JE, ed), pp 30-69. Basel: Karger.

Steriade M, Domich L, Oakson G (1986) Reticularis thamali neurons revisited: activity changes during shifts in states of vigilance. J Neurosci 6:68-81.

Tenas-Huerga N, Coll-Andreu M, Guillazo-Blanch G, Marti-Nicolovius M, Morgado-Bernal I (1998) Facilitatory effects of thalamic reticular nucleus lesions on two-way active avoidance in rats. Exp Brain Res 118:511-516.

Ward N, Brown VJ (1996) Covert orienting of attention in the rat and the role of striatal dopamine. J Neurosci 16:3082-3088.

Weese D, Brown VJ (1998) Attentional orienting in the rat is impaired by unilateral lesions in the thalamic reticular nucleus. Soc Neurosci Abstr 24:170.

Winer BJ (1971) Statistical principles in experimental design, Ed 2. New York: McGraw-Hill.

Witte EA, Davidson MC, Marrocco RT (1997) Alteration of brain catecholamine levels in Rhesus monkeys affects the alerting component of covert orienting. Psychopharmacology 132:315-323.

Yingling CD, Skinner JE (1977) Gating of thalamic input to cerebral cortex by nucleus reticularis thalami. In: Attention, voluntary contraction and event-related cerebral potentials. Progress in clinical neurophysiology, Vol 1 (Desmedt JE, ed), pp 70-96. Basel: Karger. 